\title{
Location Based Data Aggregation with Energy Aware Scheduling at RSU for Effective Message Dissemination in VANET
}

\author{
Akanksha Choudhary ${ }^{a}$, Rajeev Pandey ${ }^{\mathrm{b}}$, Anjna Deen ${ }^{\mathrm{c}}$ \\ ${ }^{a}$ Akanksha Choudhary,Student,University Institute of Technology,RGPV, Bhopal, India \\ ${ }^{b}$ Rajeev Pandey,,Assistant Professor,University Institute of Technology,RGPV,Bhopal,India \\ ${ }^{c}$ Anjna deen,Assistant Professor,University Insititute of Technology,RGPV,Bhopal,India
}

\begin{abstract}
Vehicular adhoc networks (VANETs) are relegated as a subgroup of Mobile adhoc networks (MANETs), with the incorporation of its principles. In VANET the moving nodes are vehicles which are self-administrated, not bounded and are free to move and organize themselves in the network. VANET possess the potential of improving safety on roads by broadcasting information associated with the road conditions. This results in generation of the redundant information been disseminated by vehicles. Thus bandwidth issue becomes a major concern. In this paper, Location based data aggregation technique is been proposed for aggregating congestion related data from the road areas through which vehicles travelled. It also takes into account scheduling mechanism at the road side units (RSUs) for treating individual vehicles arriving in its range on the basis of first-cum-first order. The basic idea behind this work is to effectually disseminate the aggregation information related to congestion to RSUs as well as to the vehicles in the network. The Simulation results show that the proposed technique performs well with the network load evaluation parameters.
\end{abstract}

Index Terms: Vehicular Networks, Data aggregation, Scheduling, Dissemination.

(C) 2017 Published by MECS Publisher. Selection and/or peer review under responsibility of the Research Association of Modern Education and Computer Science.

\section{Introduction}

VANETs have come up as an rapidly evolving area of research in the wireless networking domain. It comprises of vehicles as moving entities which exchange information among each other. Due to their compelling characteristics they have paved their applications in intelligent transportation systems (ITS).Its usage can be found in providing driver's safety, traffic efficiency and in other driver convenience applications based on their demands.

The practical implementation of ITS in providing efficiency in the network can be carried out by two types

* Corresponding author.

E-mail address: 
of Vehicles in the network communicate among themselves with the help of V2V communication which is governed by the OBU devices embedded in the vehicles. OBU manages to store huge amount of information at high computation speed. In V2I communication the vehicles communicate with the RSUs which are deployed at the junctions. These two types of exchanges between the communication entities help to make VANET candid and potent. The entities communicate between each other for information delivery with the help of Dedicated Short Range Communication Technology (DSRC)[1].DSRC can cover maximum upto area of $1000 \mathrm{~m}$ over the network.

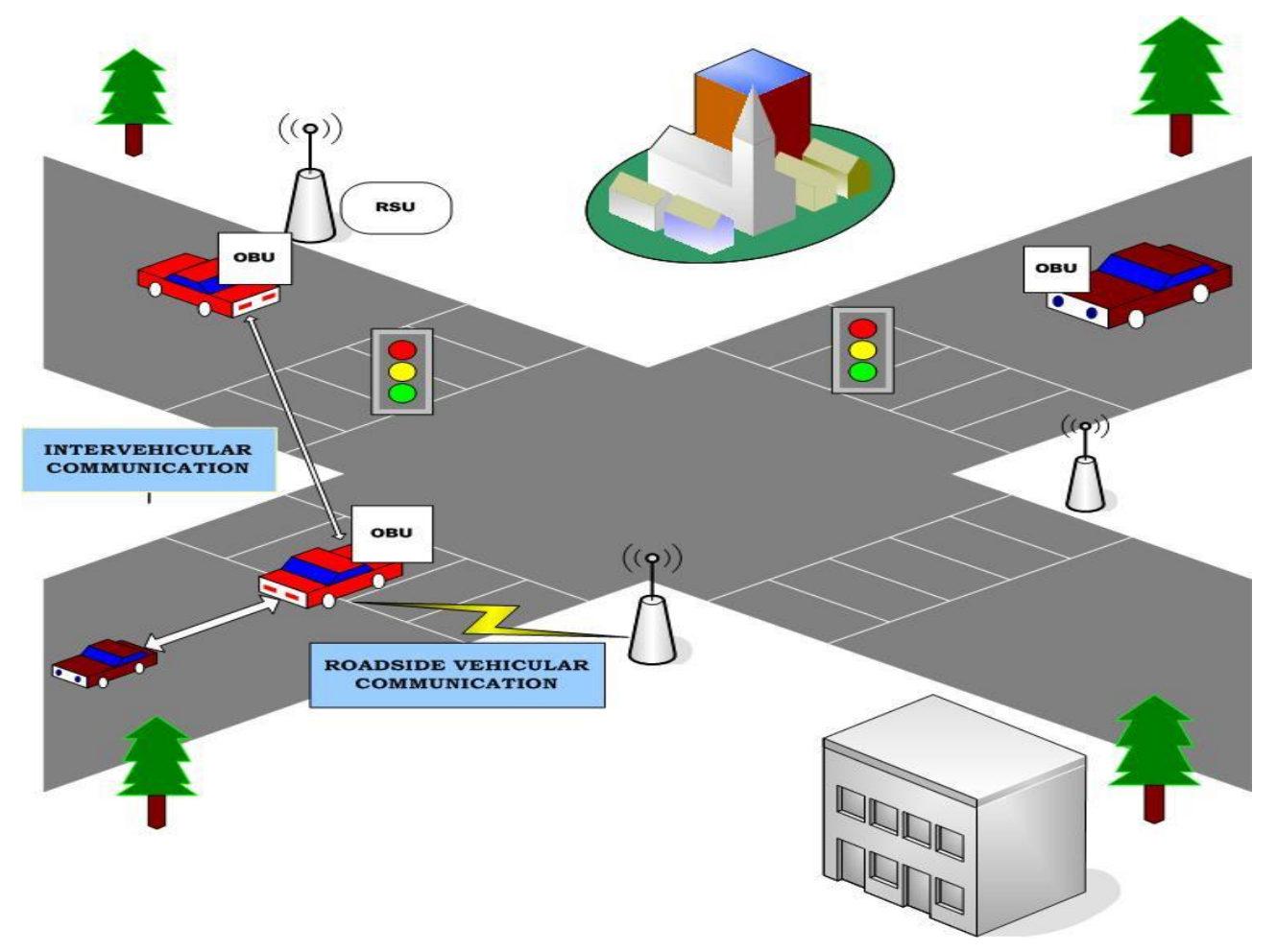

Fig.1. Ommunication Model in VANET

V2V and V2I communication[2] enables to effectively disseminate the traffic update information in the network. Each vehicle in the network generates the information related to the traffic conditions by their local observations. These leads to dissemination of the duplicate information among the vehicles. It may be possible that the observations gathered by the vehicles comprises of redundant information which may results in high bandwidth dissipation which limits the overall throughput of the network. Thus to overcome this problem our proposed technique introduces aggregation mechanism which is used for aggregating the information been received from different vehicles and then disseminating it into the network. A scheduling technique at RSU unit is also ben highlighted in this paper work. When vehicles in the network approaches towards the roadside units for retrieval or transferring information then their service order becomes essential to improve the communication efficiency. We conducted the simulation with OMNET++ simulator which demonstrates the effectiveness of our scheme.

The rest of this paper is organized as follows: The related work is given in section II. In section III, we have described our proposed methodology. Section IV represents the simulation results. Section V, describes conclusion and future work. 


\section{Related Work}

In VANETs, several data aggregation and scheduling techniques have been proposed by different authors. This section highlights some of the earlier proposed techniques

In [3] Street Smart traffic Technique is been proposed which aims at discovering and disseminating information about the congestion in VANET. It gathers accurate information about the congestion occurrence on roads. The vehicle gets the speed and its position from GPS device which is embedded within the vehicle.as the vehicle travels through the congested road sections the traffic device tracks the recordings of abnormal speeds been encountered on road. Thus it helps the vehicle to build a local map of the traffic condition and disseminate this map information to all other vehicles. This technique introduces the concept of clustering for aggregating the recodings of abnormal speed. Clustering can be used as a data aggregation technique to collect the information. Thus each vehicle keeps information about the other vehicles. This information contains the summarized details of the clusters.

In [4], Yuan Yuan et al proposed a infrastructure-free Data aggregation algorithm which is based on restricting forwarders in the network. By restricting the number of forwarders in the network the forwarders become limited which would reduce the collisions in the network. A threshold distance is used in technique for retricting the forwarders. Vehicles exchange the beacons periodically to get to know about their neighbours. When a certain vehicle upon receiving the beacon packets get to know about the vehicles who are in front and back of it within a threshold distance and they are both able to forward the information then the vehicle will mark itself as a non forwarder and if the vehicle detects the vehicle in the front and back within the distance but been marked as non forwarder then the vehicle will forward the information and will become as forwarder. Choosing proper forwarders is of great importance in the proposed technique as they help to improve the overall throughput of the network and collisions becomes less.

Christian Lochert et al in [5] VESPA approach is been introduced by the author for intelligent data aggregation. It presents a matix based approach for data aggregation, In this approach the vehicles while travelling along the roads gather some event information which is been stored in the matrix corresponding to their functional dimensions i.e location and the time at which the event was gathered. Each cell of the matrix contains an event. These aggregation matrices which contains the summary of the events information are been manipulated and are been reduced in the form of other such that the information been passed will be reliable.

In [6] a poster accurate data aggregation technique is been represented by the author which focusses on aggregation of the data by building a local map of the vehicles within a certain range and then aggregating the data described in the local map into clusters. The local map is been constructed with the help of exchanging of the primary data records which contains the speed and position of the vehicles, the signature of the vehicle and a certificate for authentication. After getting the primary data record a compact data record is been constructed by vehicles in the cluster individually. The compact data record describes the differences between the vehicle's local observations and the clusters overall record. Now aggregation is been performed by integrating the compact data records of all the vehicles in the cluster.

In [7], Bo Yu et al, proposed a Catch-up data aggregation scheme for reducing the redundancy of data. The concept introduced in the technique is how the reports from the different vehicles can be routed to the same node such that it can merged together and aggregated. Two reports are aggregated if they come from the same road sections and originate in the same time frame. For merging of reports the delay is accompassed by proposing a Markov decision model. It contains a set of actions that are intelligently performed by the vehicles. These set of actions include two main functions i.e WALK and RUN. Short delay is performed by the RUN action and longer delay is performed by the WALK fucntion.

In [8] Caliskan et al, proposed a aggregation technique which disseminated the parking information to the vehicles. Quadtree construction is been carried out which divides the city into non overlying cells. With every higher level cell, four lowest level cells are combined to form one higher level cell. The basic objective is to 
broadcast more accurate information about free parking zones in local area and only distribute obscene information in larger areas. The vehicles periodically aggregates the vacant parking spaces whenever information of all four framework cells is available.

Milos Milojevic in [9] proposed a location based data aggregation technique which aggregates the information of the traversed road areas and effciently disseminate the information into the network by adaptive broadcasting.

Various scheduling mechanisms are also been proposed by the authors in previous works to improve the communication effciency.

In [10] Yang Zhang et al. proposed Service Scheduling of Vehicle-Roadside Data Access approach which presents a service scheduling scheme for treating vehicles which enters the radio coverage range of RSU. The service scheduling scheme is based on two parameters: -Deadline and Data size. Two techniques are been proposed i.e First deadline first (FDF) and smallest Data size first(SDF).In first deadline first approach the vehicle whose request is urgent is been served first. The assumption is made that each vehicle knows its deadline of request. This is because when a vehicle equippedget to know about the tota with a GPS device comes under the coverage area of a RSU, it can estimate its departure time based on knowledge of its driving velocity and its geographic location. The technique proposed can make the vehicle download or upload the data to the RSU.

In [11] the author presented a Energy-Efficient Roadside Unit Scheduling for Maintaining Connectivity in VANET. This technique aims at enhancing the energy efficiency of the deployed RSU along the roads by optimal scheduling which turns them off and on when not in use within a given period of time. This saves the energy of road-side units. Snapshot scheduling and snapshot selection approaches are been used to implement this technique in network. Snapshot scheduling decides the minimum number of Road-side units that are required for the snapshot that will be created for VANET at a certain time, whilst the snapshot selection problem determines the time points sequence at which the snapshot should be updated.

Based on the above proposed techniques, the communication effciency can be enhanced by effciently disseminating the aggregated information with the help of centralised infrastructures.

\section{Proposed Work}

In our propose methodology, for minimizing the communication overhead we proposed a location based data aggregation mechanism which aggregates the data based on interpacket delay between the vehicles. Beacon signals are periodically exchanged among the vehicles. Thus from the acknowledgement of the beacon signals the vehicles get to know about the exact total time taken for the packet to reach the destination. If the interpacket delay becomes greater than the total time taken there arises the congestion. This information is then aggregated and disseminated to other vehicles and RSUs which in turn will forward the messages to other RSUs.

If the packet is sent to the same vehicle whose total time of beacon acknowledgement is been recorded then interpacket delay is defined as the difference of the time taken by the packet to reach the destination to the sending time of the packet to reach the destination. The beacon signals exchanged between the vehicles contain the vehicle ID, speed and location of the vehicle. By aggregating the traffic related data of the specified locations through which the vehicles travelled, the redundancy generation of the information is greatly reduced which leads to increase the efficiency of the network. The static nodes i.e RSUs are been deployed in our scheme for disseminating of the messages over large distances. Vehicles coming under the range of RSU transfers the congestion related aggregated data to it. It may be possible that at a given point of time many vehicle try to simultaneously disseminate the data to RSU which would result in high load. Thus we proposed scheduling technique for giving priority to vehicle for disseminating information. Vehicles are treated on firstcum-first order. The vehicle arriving firstly under the range of RSU it will be given time slot first based on nonpreemption basis. 


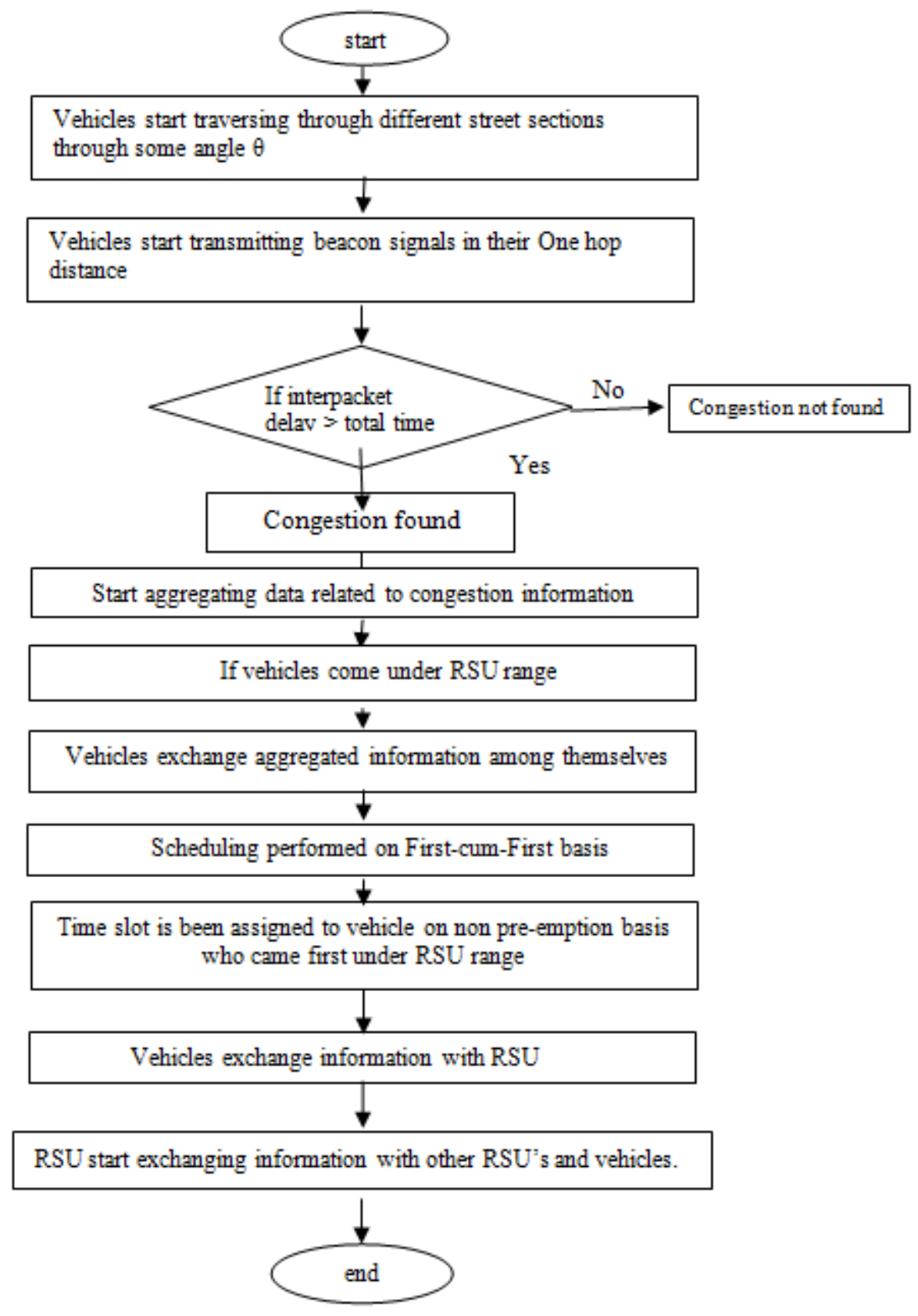

Fig.2. Proposed Technique Workflow

\section{Simulation and Results}

The performance evaluation of the proposed work is done with the help of OMNET++ simulator whihc is 
open source software. In results analysis following simulation parameters were used. To evaluate the performance of our proposed technique we compared the results with the results of the reference simulation i.e Location based data aggregation for efficient message dissemination in VANET.

Table 1. Simulation Parameters

\begin{tabular}{|c|c|}
\hline Road Traffic Density & Low \\
\hline No of vehicles & 250 \\
\hline Total simulation area & $10000 \mathrm{~m} * 10000 \mathrm{~m}$ \\
\hline Simulation time & $200 \mathrm{sec}$ \\
\hline Communication standard & IEEE $802.11 \mathrm{p}$ \\
\hline Tx power & $20 \mathrm{~mW}$ \\
\hline Rx sensitivity & $-94 \mathrm{dBm}$ \\
\hline MAC bit rate & $18 \mathrm{Mbps}$ \\
\hline
\end{tabular}

To evaluate the performance of the proposed technique on the network load we overviewed our proposed technique based average number of sent messsages, received messages, the number of vehicles that went into backoff procedure and the number of lost packets in the network.

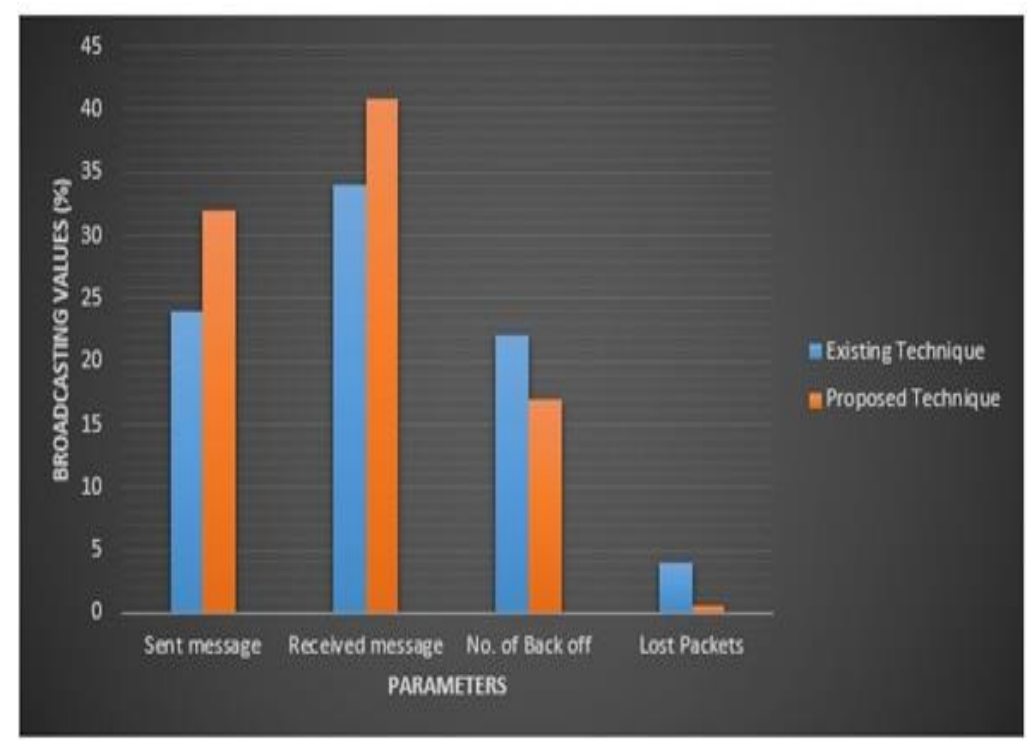

Fig.3. Network Load Evaluation of Existing Technique(LA) And Proposed Technique Showing Average Values Per Vehicle In Simulation.

\subsection{Packet Delivery Ratio:-}

Packet delivery ratio is the ratio of number of messages received in the network to the number of messages been sent in the network. 


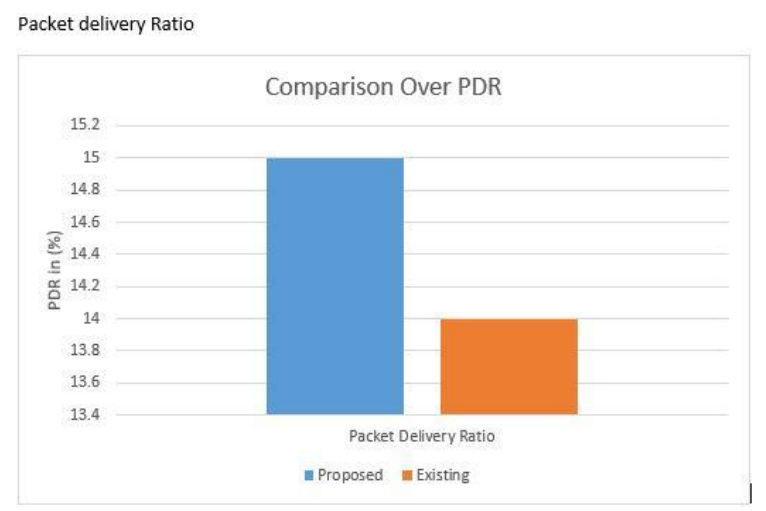

Fig.4. Packet delivery Ratio Analysis

Comparison of the results of the proposed technique performs significantly better than the existing technique[9].

\section{Conclusion}

In this work we focussed on the data aggregation stategy by the vehicles for broadcasting of the congestion related information. In our future work we will be focussing on mechanisms which are suitable for the dissemination of any other generic information in the network like accident related information or information related to parking places on the roads. Additionally the scheduling mechanism been applied at the RSU unit can be examined under high density of vehicles and can be enhanced accordingly to perform well in high load conditions. The first-cum-first serve scheduling can be used in accidental situations also. The vehicles which contain accidental information are treated firstly for transfer of information to RSU.

\section{References}

[1] Liang, Wenshuang, et al. "Vehicular ad hoc networks: Architectures, research issues, challenges and trends." International Conference on Wireless Algorithms, Systems, and Applications. Springer International Publishing, 2014.

[2] S. Zeadally, R. Hunt, Y.S. Chen, "Vehicular ad hoc networks (VANETS): status, results, and challenges," Telecommunication Systems, Springer, 2010.

[3] Dornbush, Sandor, and Anupam Joshi. "StreetSmart traffic: Discovering and disseminating automobile congestion using VANET's." 2007 IEEE 65th Vehicular Technology Conference-VTC2007-Spring. IEEE, 2007.

[4] Yuan, Yuan, et al. "DA2RF: a data aggregation algorithm by restricting forwarders for VANETs." Computing, Networking and Communications (ICNC), 2014 International Conference on. IEEE, 2014.

[5] Defude, Bruno, et al. "Data aggregation in VANETs: the VESPA approach. "Proceedings of the 5th Annual International Conference on Mobile and Ubiquitous Systems: Computing, Networking, and Services. ICST (Institute for Computer Sciences, Social-Informatics and Telecommunications Engineering), 2008.

[6] Ibrahim, Khaled, and Michele C. Weigle. "Accurate data aggregation for VANETs." Proceedings of the fourth ACM international workshop on Vehicular ad hoc networks. ACM, 2007. 
[7] $\mathrm{Yu}, \mathrm{Bo}$, Jiayu Gong, and Cheng-Zhong Xu. "Catch-up: a data aggregation scheme for vanets." Proceedings of the fifth ACM international workshop on VehiculAr Inter-NETworking. ACM, 2008.

[8] Caliskan, Murat, Daniel Graupner, and Martin Mauve. "Decentralized discovery of free parking places." Proceedings of the 3rd international workshop on Vehicular ad hoc networks. ACM, 2006.

[9] Milojevic, Milos, and Veselin Rakocevic. "Location Aware Data Aggregation for Efficient Message Dissemination in Vehicular Ad Hoc Networks." IEEE Transactions on Vehicular Technology 64.12 (2015): 5575-5583

[10] Zhang, Yang, Jing Zhao, and Guohong Cao. "Service scheduling of vehicle-roadside data access." Mobile Networks and Applications 15.1 (2010): 83-96.

[11] Zou, Feng, et al. "Energy-efficient roadside unit scheduling for maintaining connectivity in vehicle ad-hoc network." Proceedings of the 5th International Conference on Ubiquitous Information Management and Communication. ACM, 2011

[12] Berlin, M. A., and Sheila Anand. "Driving hazards message propagation using road side infrastructure in VANETs." International Conference on Computer Science and Information Technology. Springer Berlin Heidelberg, 2012.

[13] Kadakia, Ruchit, Akshay Bhatt, and Lakshmi Kurup. "A Review Paper of Data Aggregation in VANET Architecture." International Journal of Current Engineering and Technology (2014).

[14] Kumar, Rakesh, and Mayank Dave. "A review of various vanet data dissemination protocols." International Journal of u-and e-Service, Science and Technology 5.3 (2012): 27-44.

[15] Cseh C, "Architecture of the dedicated short-range communications (DSRC) protocol," In: Proceedings of. IEEE vehicular technology conference, VTC; 1998.

\section{Authors' Profiles}

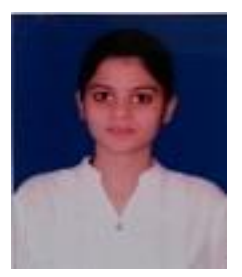

Ms. Akanksha Choudhary: Ms. Choudhary is currently pursuing Integrated Dual Degree Course(BE+MTECH) in Computer science \& Engineering from UITRGPV, Bhopal, India. Her research areas are Computer Networks, Vehicular technology and advancements, Efficient communication in Vehicular Adhoc Networks.

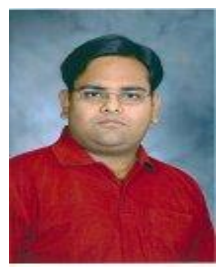

Dr. Rajeev Pandey: Dr. Pandey received his Bachelor's Degree in Computer Science and Engineering in 2001 from IET, Agra(IN). M.E. (Computer Science and Engineering) in 2004 from Dr. B.R.A. University, Agra(IN). Ph.D. in 2010 from Dr. B.R.A University, Agra(IN). $\mathrm{He}$ is also pursuing Ph.D. (Computer Science and Engineering) from RGPV University, Bhopal(IN).At present, he is working as an Assitant Professor in Department of Computer Science and Engineering, UIT-RGPV, Bhopal Since July 2007. 


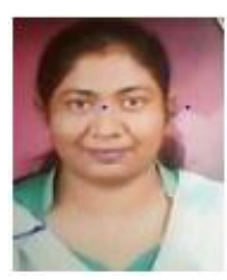

Assistant Professor Anjna Deen: Mrs. Deen has received her Bachelor's degree in Computer Science \& Engineering from UIT (GEC)-RGPV, Bhopal India in 1993, and completed M.Tech. degree from Maulana Azad National Institute of Technology, Bhopal, India in 2007. At present, she is working as an Assistant Prof. in UIT-RGPV Bhopal since 1996 .She has published various research papers in National and International Journals. Her areas of interest include Computer Networks, Neural Networks, Wireless communication and Bioinformatics.

How to cite this paper: Akanksha Choudhary, Rajeev Pandey, Anjna Deen,"Location Based Data Aggregation with Energy Aware Scheduling at RSU for Effective Message Dissemination in VANET", International Journal of Engineering and Manufacturing(IJEM), Vol.7, No.3, pp.49-57, 2017.DOI: 10.5815/ijem.2017.03.06 